\title{
Calculation of Nucleon Electric Dipole Moments Induced by Quark Chromo-Electric Dipole Moments and the QCD $\theta$-term
}

\author{
Sergey Syritsyn ${ }^{* a, b}$ Taku Izubuchi $^{b}$, Hiroshi Ohki ${ }^{a, c}$, \\ ${ }^{a}$ RIKEN/BNL Research Center, Brookhaven National Laboratory, Upton, NY 11973, USA \\ ${ }^{b}$ Department of Physics and Astronomy, Stony Brook University, Stony Brook, NY 11794, USA \\ ${ }^{c}$ Department of Physics, Nara Women's University, Nara 630-8506, Japan \\ E-mail: ssyritsyn@quark phy ·bnl ·gov
}

\begin{abstract}
Electric dipole moments (EDMs) of nucleons and nuclei, which are sought as evidence of CP violation, require lattice calculations to connect constraints from experiments to limits on the strong $\mathrm{CP}$ violation within QCD or CP violation introduced by new physics from beyond the standard model. Nucleon EDM calculations on a lattice are notoriously hard due to large statistical noise, chiral symmetry violating effects, and potential mixing of the EDM and the anomalous magnetic moment of the nucleon. In this report, details of ongoing lattice calculations of proton and neutron EDMs induced by the QCD $\theta$-term and the quark chromo-EDM, the lowest-dimension effective $\mathrm{CP}$-violating quark-gluon interaction are presented. Our calculation employs chiral-symmetric fermion discretization. An assessment of feasibility of nucleon EDM calculations at the physical point is discussed.
\end{abstract}

36th annual International Symposium on Lattice Field Theory

22-28 July 2018

Michigan State University, USA

${ }^{*}$ Speaker. 


\section{Note:}

The material associated with this conference contribution is identical to the material presented at the "XIIIth Quark Confinement and the Hadron Spectrum" conference (Confinement2018), Maynooth University, Ireland, 31 July - 6 August 2018, and published in the proceedings of that conference. Rather than reproducing a duplicate of the XIIIth Confinement proceedings contribution here, the reader is referred to those proceedings:

S. Syritsyn, T. Izubuchi, H. Ohki, "Calculation of Nucleon Electric Dipole Moments Induced by Quark Chromo-Electric Dipole Moments and the QCD $\theta$-term",

in: Proceedings of the XIIIth Quark Confinement and the Hadron Spectrum conference (Confinement2018), Maynooth University, Ireland, 31 July - 6 August 2018, PoS Confinement2018 194.

\section{ACKNOWLEDGEMENTS}

We are grateful for the gauge configurations provided by the RBC/UKQCD collaboration. This research used resources of the Argonne Leadership Computing Facility, which is a DOE Office of Science User Facility supported under Contract DE-AC02-06CH11357, and Hokusai supercomputer of the RIKEN ACCC facility. SS is supported by the RHIC Physics Fellow Program of the RIKEN BNL Research Center. TI is supported in part by US DOE Contract DESC0012704(BNL), and JSPS KAKENHI grant numbers JP26400261, JP17H02906. HO is supported in part by JSPS KAKENHI Grant Numbers 17K14309. 